\title{
Editorial: A Message from the New Editor-in-Chief
}

I am honored to be serving as the new Editor-in-Chief of ACM Transactions on Design Automation of Electronic Systems (TODAES). TODAES, under the leadership of Professor Naehyuck Chang, the EiC over the past six years, and with all the hard work of its AEs and reviewers, has firmly established itself as a premier ACM journal in electronic design automation (EDA). TODAES now publishes leading research results covering a wide spectrum of topics in the EDA field with historically short review periods. My goal is to build an even stronger TODAES community.

Having worked in the field of EDA for more than 25 years, I believe that now is an opportune time for TODAES to reinvigorate itself by addressing the challenges that it faces. Besides maintaining the short review cycles that TODAES has successfully achieved, I will target three specific challenges: (i) growing the scope of the journal without losing its focus, (ii) increasing the number of high-quality submissions, and (iii) improving the impact factor.

Compared to many other ACM journals, TODAES is a truly interdisciplinary publication, as research findings published in TODAES not only enable but also are stimulated by advances in both computer science and electronic systems. The recent rise of AI applications, edge computing paradigms, and emerging architectures and technologies induces fundamentally different challenges to the EDA field and calls for innovations in electronic system design and design automation. The EDA community is well positioned to be the driver of these innovations, and TODAES is a key publication venue for these innovations. However, it is important that TODAES continues to focus on design- and design-automation-related topics to remain a journal with a unique identification. To address this challenge, I have updated the scope of TODAES to encompass emerging research topics in design and design automation. (See the updated scope at https://dl.acm.org/journal/todaes/about.) Furthermore, TODAES will actively solicit special issues, surveys, and tutorials in the new topic areas. I specifically encourage more special issues and regular submissions on interdisciplinary and cross-layer topics.

Increasing the number of high-quality submissions will further enhance the overall quality of TODAES and its status as the premier ACM journal in EDA. Toward this goal, I have introduced two new types of articles. One is the Keynote article, which will provide perspectives in terms of the state-of-the-art development and future directions on specific topics. The other is the Designer Note short article, in which practitioners will share their design experiences, challenges, and trends. (More details about the manuscript types can be found at https:// dl.acm.org/journal/todaes/author-guidelines.) More article types may be introduced later. To ensure sustained high-quality articles in these categories, I will establish a special AE group that will specifically focus on soliciting articles for these categories.

Though impact factor is not an absolute metric to measure the quality of a journal, especially when comparing journals across different research fields, it does reflect the degree of recognition

\footnotetext{
ACM Reference format: Syst. 25, 6, Article 49e (September 2020), 2 pages.

https://doi.org/10.1145/3419376

(C) 2020 Copyright held by the owner/author(s).

1084-4309/2020/09-ART49e

https://doi.org/10.1145/3419376
}

X. Sharon Hu. 2020. Editorial: A Message from the New Editor-in-Chief. ACM Trans. Des. Autom. Electron.

ACM Transactions on Design Automation of Electronic Systems, Vol. 25, No. 6, Article 49e. Pub. date: September 2020 
of the published articles in the journal within the respective research field. Successfully addressing the first two challenges above is fundamental and will improve the impact factor of TODAES. I also plan to take specific steps to increase the awareness of the articles published in TODAES. For example, I will ask AEs to work with the authors to ensure that the abstract of each published article clearly states the impact of the work. I will actively promote top-cited and top-downloaded articles, not only on the TODAES website but also through news items in the SIGDA Newsletter and other channels. Furthermore, I will devote time and effort to help increase the international visibility and reputation of TODAES through targeted promotional activities.

In conclusion, taking TODAES to the next level will require teamwork. I welcome suggestions from the TODAES authors and readers as well as the larger community and will provide an online form for this. Together we will solidify TODAES' position as a premier publication in electronic system design and automation!

X. Sharon $\mathrm{Hu}$ 